\title{
Stability analysis of a fractional-order two-species facultative mutualism model with harvesting
}

\author{
Nattakan Supajaidee ${ }^{1}$ and Sompop Moonchai ${ }^{2,3^{*}}$
}

\author{
"Correspondence: \\ tumath@gmail.com \\ ${ }^{2}$ Center of Excellence in \\ Mathematics and Applied \\ Mathematics, Department of \\ Mathematics, Faculty of Science, \\ Chiang Mai University, Chiang Mai, \\ 50200, Thailand \\ ${ }^{3}$ Centre of Excellence in \\ Mathematics, CHE, 328 Si Ayutthaya \\ Road, Bangkok, Thailand \\ Full list of author information is \\ available at the end of the article
}

\begin{abstract}
We present a fractional-order model of two-species facultative mutualism with harvesting. We investigate the stability of the equilibrium points of the model by using the linearization method for noncoexistence of equilibrium points and the Lyapunov direct method for the positive coexistence of an equilibrium point. In addition, we obtain sufficient conditions to ensure the local asymptotic stability and global uniform asymptotic stability for the model. Finally, we provide illustrated numerical examples to verify the stability results obtained in this study.
\end{abstract}

Keywords: fractional differential equations; stability analysis; mutualism model; Caputo fractional derivative; Lyapunov function

\section{Introduction}

A population model is a mathematical model used to describe population dynamics. Equations describing population models can take different forms such as difference equations $[1,2]$, differential equations [1, 2], or delay differential equations [3]. There are several types of population models such as interspecific competition models, predator-prey models, and facultative mutualism models [4].

Two-species facultative cooperative interaction or facultative mutualism is the interaction between two different biological species in which both species benefit [5], such as herbivorous crabs and coralline algae [6]. The famous two-species mutualism models are derived from modified Lotka-Volterra competition equations [7] and have been applied to a variety of ecological interactions [8-11]. In 2012, Legovic and Gecek [12] expanded the mutualism model by adding a harvesting effort specific to the population. The model is described by a system of first-order differential equations of the form

$$
\left\{\begin{array}{l}
\frac{d x_{1}}{d t}=\widehat{r}_{1} x_{1}\left(1-\frac{\widehat{e}_{1}}{\widehat{r}_{1}}\right)-\frac{\widehat{1}_{1} x_{1}^{2}}{\widehat{K}_{1}+\widehat{b}_{12} x_{2}}, \\
\frac{d x_{2}}{d t}=\widehat{r}_{2} x_{2}\left(1-\frac{\widehat{e}_{2}}{\widehat{r}_{2}}\right)-\frac{\widehat{r}_{2} x_{2}^{2}}{\widehat{K}_{2}+\widehat{b}_{21} x_{1}},
\end{array}\right.
$$

where all the parameters are positive constants, $x_{i}$ denotes the population density of the $i$ th species at time $t, \widehat{r}_{i}$ is the intrinsic birth rate of the $i$ th species, $\widehat{K}_{i}$ is the carrying capacity of the environment, and $\widehat{e}_{i}$ is the harvesting effort upon the same species $x_{i}$ for $i=1,2$. In

(c) The Author(s) 2017. This article is distributed under the terms of the Creative Commons Attribution 4.0 International License (http://creativecommons.org/licenses/by/4.0/), which permits unrestricted use, distribution, and reproduction in any medium, provided you give appropriate credit to the original author(s) and the source, provide a link to the Creative Commons license, and indicate if changes were made. 
addition, $\widehat{b}_{12}$ and $\widehat{b}_{21}$ represent the extent of cooperative relationships of $x_{1}$ and $x_{2}$, that is, the mutualistic support the species give each other.

This system is an illustration of the situation in which the effects of mutualism have the most impact when the recipient population is at high density (Wolin and Lawlor [13]). If one species is missing, then the dynamics of the other species is characterized by logistic growth [14].

In recent years, fractional calculus has become an intriguing field. There are several definitions of the fractional-order derivative. Among them, the definitions commonly used are the Riemann-Liouville definition, the Grünwald-Letnikov definition [15], and the Caputo definition [16]. The Caputo derivative is reformulated from the more classic RiemannLiouville derivative, and the initial conditions for Caputo fractional differential equations are expressed in the same manner as for integer-order differential equations [15]. For this reason, in this paper, we use the Caputo derivative. This is likely to be a good choice to solve problems in the dynamics of complex systems because the order of derivatives can be any real or complex number. Especially, the results may more closely resemble realistic dynamics than integer-order systems [17]. The advantages of fractional calculus support its use in applied mathematics, physics, chemistry, engineering, and even finance and social sciences [18]. Some examples include data-fitting problems for blood alcohol level, video tape counter readings and models for world population growth [19], obesity epidemics [20], Ebola epidemics [21], and bovine babesiosis disease and tick populations [22].

Stability analysis is an important tool to understand system dynamics. Recently, there have been many studies concerning the stability and asymptotic behavior of models in the form of differential equations [23-26], delay differential equations [27-30], and fractional differential equations [31-35].

In the case of fractional differential equations, linearization and Lyapunov methods have been a popular technique to analyze the stability of a system without visibly solving the equations [36-38]. The linearization method is applied to approximate the nonlinear system by using the linearized model $[36,39]$. On the other hand, the Lyapunov method is used to investigate the stability of an equilibrium point of a system based on a Lyapunov function. In recent years, many researchers proposed a Lyapunov direct method to analyze the stability of fractional differential equations [37, 40-44]. Moreover, some Lyapunov functions have been constructed to study the stability of fractional differential equations [45-48]. However, the construction of a Lyapunov function and calculation of the fractional derivatives is complicated $[42,44]$.

In this paper, we present a fractional model for two-species facultative mutualism. Moreover, we analyze the stability of the equilibrium points for this model to obtain sufficient conditions for the local asymptotic stability of the noncoexistence equilibrium points using the linearization method. Conversely, we obtain sufficient conditions for the global uniformly asymptotic stability of the coexistence equilibrium point using the Lyapunov method.

We organize the paper as follows. In Section 2, we give some necessary definitions and some known properties. In Section 3, we propose a fractional-order two-species facultative mutualism model. In Section 4, we investigate the local stability of the three noncoexistence equilibrium points and the global stability of the coexistence equilibrium point in the sense of Lyapunov for the proposed model. Some numerical simulations to validate the 
theoretical results are shown in Section 5. In the last section, we present the conclusions of this paper.

\section{Preliminaries}

In this section, we introduce some definitions of fractional calculus and several important theorems of stability analysis.

Definition 2.1 ([15]) For a function $f \in C^{n}$ given on the interval $\left[t_{0}, \infty\right]$, suppose that $\alpha>0$ and $t>t_{0}$, where $\alpha, t_{0}, t \in \mathbb{R}$, and $n \in \mathbb{N}$. Then

$$
{ }_{t_{0}}^{C} D_{t}^{\alpha} f(t)= \begin{cases}\frac{1}{\Gamma(n-\alpha)} \int_{t_{0}}^{t} \frac{f^{(n)}(\tau)}{(t-\tau)^{\alpha+1-n}} d \tau, & n-1<\alpha<n, \\ \frac{d^{(n)}}{d t^{(n)}} f(t), & \alpha=n,\end{cases}
$$

is called the Caputo fractional derivative of order $\alpha$, where $\Gamma$ is the gamma function.

Remark 2.2 ([15]) Under natural conditions on the function $f(t)$, as $\alpha \rightarrow n$, the Caputo derivative becomes the conventional $n$th derivative of the function $f(t)$.

Remark 2.3 ([45]) The Caputo fractional derivative of order $0<\alpha<1$ for a smooth function $f=f(t)$ is given by

$$
{ }_{t_{0}}^{C} D_{t}^{\alpha} f(t)=\frac{1}{\Gamma(1-\alpha)} \int_{t_{0}}^{t} \frac{1}{(t-\tau)^{\alpha}} \frac{d f(\tau)}{d \tau} d \tau
$$

Theorem 2.4 ([49]) Let $\alpha>0, n-1<\alpha<n \in \mathbb{N}$. Suppose that $f(t), f^{\prime}(t), \ldots, f^{(n-1)}(t)$ are continuous on $\left[t_{0}, \infty\right)$ and of exponential order and that ${ }_{t_{0}}^{C} D_{t}^{\alpha} f(t)$ is piecewise continuous on $\left[t_{0}, \infty\right)$. Then

$$
\mathcal{L}\left\{{ }_{t_{0}}^{C} D_{t}^{\alpha} f(t)\right\}=s^{\alpha} F(s)-\sum_{k=0}^{n-1} s^{\alpha-k-1} f^{(k)}\left(t_{0}\right),
$$

where $F(s)=\mathcal{L}\{f(t)\}$.

Theorem 2.5 ([50]) Let $C$ be the complex plane. For any $\alpha>0, \beta>0$, and $A \in \mathbb{C}^{n \times n}$, we have

$$
\mathcal{L}\left\{t^{\beta-1} E_{\alpha, \beta}\left(A t^{\alpha}\right)\right\}=s^{\alpha-\beta}\left(s^{\alpha}-A\right)^{-1}
$$

for $\mathcal{R} s>\|A\|^{\frac{1}{\alpha}}$, where $\mathcal{R} s$ represents the real part of the complex number $s$, and $E_{\alpha, \beta}$ is the Mittag-Leffler function [15].

Theorem 2.6 ([37]) Consider the fractional-order system

$$
{ }_{t_{0}}^{C} D_{t}^{\alpha} x(t)=f(t, x)
$$

with initial condition $x\left(t_{0}\right)$, where $\alpha \in(0,1), f:\left[t_{0}, \infty\right) \times \Omega \rightarrow \mathbb{R}^{n}$ is piecewise continuous in t and locally Lipschitz in $x$ on $\left[t_{0}, \infty\right) \times \Omega$, and $\Omega \subseteq \mathbb{R}^{n}$ is a domain containing the origin 
$x=0$. The constant $x^{*}$ is an equilibrium point of the Caputo fractional-order system (2.3) if and only if $f\left(t, x^{*}\right)=0$.

Definition 2.7 ([51]) For the system described by (2.3):

(i) The trivial solution is said to be stable if for any $t_{0} \in \mathbb{R}$ and any $\varepsilon>0$, there exists $\delta=\delta\left(t_{0}, \varepsilon\right)>0$ such that $\left\|x\left(t_{0}\right)\right\|<\delta$ implies $\|x(t)\|<\varepsilon$ for all $t>t_{0}$.

(ii) The trivial solution is said to be asymptotically stable if it is stable and for any $t_{0} \in \mathbb{R}$ and any $\varepsilon>0$, there exists $\delta_{a}=\delta_{a}\left(t_{0}, \varepsilon\right)>0$ such that $\left\|x\left(t_{0}\right)\right\|<\delta_{a}$ implies $\lim _{t \rightarrow \infty}\|x(t)\|=0$.

(iii) The trivial solution is said to be uniformly stable if it is stable and $\delta=\delta(\varepsilon)>0$ can be chosen independently of $t_{0}$.

(iv) The trivial solution is uniformly asymptotically stable if it is uniformly stable and there exists $\delta_{a}>0$, independent of $t_{0}$, such that if $\left\|x\left(t_{0}\right)\right\|<\delta_{a}$, then $\lim _{t \rightarrow \infty}\|x(t)\|=0$.

(v) The trivial solution is globally (uniformly) asymptotically stable if it is (uniformly) asymptotically stable and $\delta_{a}$ can be an arbitrary large finite number.

Theorem 2.8 ([34]) The equilibrium points $x^{*}$ of system (2.3) are locally asymptotically stable if all eigenvalues $\lambda_{i}$ of the Jacobian matrix $J=\frac{\partial f}{\partial x}$ evaluated at the equilibrium points satisfy $\left|\arg \left(\lambda_{i}\right)\right|>\frac{\alpha \pi}{2}$.

Theorem 2.9 (Uniform Asymptotic Stability Theorem [42]) Let $x=0$ be an equilibrium point of system (2.3), and let $\Omega \subset \mathbb{R}^{n}$ be a domain containing $x=0$. Let $V(t, x):\left[t_{0}, \infty\right) \times$ $\Omega \rightarrow \mathbb{R}$ be a continuously differentiable function such that

$$
W_{1}(x) \leq V(t, x) \leq W_{2}(x)
$$

and

$$
{ }_{t_{0}}^{C} D_{t}^{\alpha} V(t, x) \leq-W_{3}(x)
$$

for $t \geq 0, x \in \Omega$, and $0<\alpha<1$, where $W_{1}(x), W_{2}(x)$, and $W_{3}(x)$ are continuous positive definite functions on $\Omega$. Then $x=0$ is uniformly asymptotically stable.

Remark 2.10 ([37]) If $x=x^{*}$ is the equilibrium point of system (2.3) and satisfies the conditions of Theorem 2.9 , then $x=x^{*}$ is uniformly asymptotically stable.

Theorem 2.11 ([45]) Let $x(t) \in \mathbb{R}^{+}$be a continuous and derivable function. Then, for any time instant $t \geq t_{0}$ and all $\alpha \in(0,1)$,

$$
{ }_{t_{0}}^{C} D_{t}^{\alpha}\left(x(t)-x^{*}-x^{*} \ln \frac{x(t)}{x^{*}}\right) \leq\left(1-\frac{x(t)}{x^{*}}\right){ }_{t_{0}}^{C} D_{t}^{\alpha} x(t)
$$

where $x^{*} \in \mathbb{R}^{+}$.

\section{Model description}

We construct the proposed model for fractional-order two-species facultative mutualism by improving system (1.1). To do so, we replace the integer-order derivatives with 
fractional-order Caputo-type derivatives; the resulting equation is written as

$$
\left\{\begin{array}{l}
{ }_{t_{0}}^{C} D_{t}^{\alpha} x_{1}=\widehat{r}_{1} x_{1}\left(1-\frac{\widehat{e}_{1}}{\widehat{r}_{1}}\right)-\frac{\widehat{r}_{1} x_{1}^{2}}{\widehat{K}_{1}+b_{12} x_{2}}, \\
{ }_{t_{0}}^{C} D_{t}^{\alpha} x_{2}=\widehat{r}_{2} x_{2}\left(1-\frac{\widehat{e}_{2}}{\widehat{r}_{2}}\right)-\frac{\widehat{r}_{2} x_{2}^{2}}{\widehat{K}_{2}+\widehat{b}_{21} x_{1}}
\end{array} .\right.
$$

System (3.1) has some flaws as regards the time dimension because the left-hand side has the dimension $(\text { time })^{-\alpha}$, whereas the right-hand side has the dimension $(\text { time })^{-1}$. The correct form of system (3.1) can be obtained as follows [52]:

$$
\left\{\begin{array}{l}
{ }_{t_{0}}^{C} D_{t}^{\alpha} x_{1}=\widehat{r}_{1}^{\alpha} x_{1}\left(1-\frac{\widehat{e}_{1}^{\alpha}}{\widehat{r}_{1}^{\alpha}}\right)-\frac{\widehat{r}_{1}^{\alpha} x_{1}^{2}}{\widehat{K}_{1}+\widehat{b}_{12} x_{2}}, \\
{ }_{t_{0}}^{C} D_{t}^{\alpha} x_{2}=\widehat{r}_{2}^{\alpha} x_{2}\left(1-\frac{\widehat{e}_{2}^{\alpha}}{\widehat{r}_{2}^{\alpha}}\right)-\frac{\widehat{r}_{2}^{\alpha} x_{2}^{2}}{\widehat{K}_{2}+\widehat{b}_{21} x_{1}}
\end{array} .\right.
$$

For convenience, we define the parameters $r_{i}=\widehat{r}_{i}^{\alpha}, e_{i}=\widehat{e}_{i}^{\alpha}, K_{i}=\widehat{K}_{i}, b_{12}=\widehat{b}_{12}$, and $b_{21}=\widehat{b}_{21}$, where $i=1,2$. Then, we obtain the following modified system:

$$
\left\{\begin{array}{l}
{ }_{t_{0}}^{C} D_{t}^{\alpha} x_{1}=f_{1}\left(x_{1}, x_{2}\right)=r_{1} x_{1}\left(1-\frac{e_{1}}{r_{1}}\right)-\frac{r_{1} x_{1}^{2}}{K_{1}+b_{12} x_{2}}, \\
{ }_{t_{0}}^{C} D_{t}^{\alpha} x_{2}=f_{2}\left(x_{1}, x_{2}\right)=r_{2} x_{2}\left(1-\frac{e_{2}}{r_{2}}\right)-\frac{r_{2} x_{2}^{2}}{K_{2}+b_{21} x_{1}},
\end{array}\right.
$$

with initial conditions $x_{1}\left(t_{0}\right)=x_{10}$ and $x_{2}\left(t_{0}\right)=x_{20}$, where all the model parameters are assumed to be positive.

\subsection{Existence and uniqueness}

The possible region of model (3.3) is defined as the nonnegative quadrant

$$
\mathbb{R}_{+}^{2}=\left\{x=\left(x_{1}, x_{2}\right) \in \mathbb{R}^{2}: x_{1} \geq 0, x_{2} \geq 0\right\} .
$$

From system (3.3) it is clear that $f_{i}$ and $\frac{\partial f_{i}}{\partial x_{j}}$ for $i=1,2$ and $j=1,2$ are continuous in $\mathbb{R}_{+}^{2}$. Following the lemma in [53], we can conclude that $f=\left(f_{1}, f_{2}\right)$ satisfies the local Lipschitz condition with respect to $x=\left(x_{1}(t), x_{2}(t)\right)$ in $\mathbb{R}_{+}^{2}$. Therefore, by Remark 3.8 in [15], system (3.3) has a unique solution in $\mathbb{R}_{+}^{2}$.

\subsection{Nonnegative solution}

Theorem 3.1 If $x_{1}\left(t_{0}\right) \geq 0$ and $x_{2}\left(t_{0}\right) \geq 0$, then there is a unique solution $x(t)$ to the Caputo fractional-order model (3.3) on $t \geq t_{0}$, and the solution remains in $\mathbb{R}_{+}^{2}$.

Proof In Section 3.1, the uniqueness of a solution of $x(t)$ to system (3.3) is obtained. Thus, we only need to prove that the solution $x(t)=\left(x_{1}(t), x_{2}(t)\right)$ remains in $\mathbb{R}_{+}^{2}$.

Let $x\left(t_{0}\right)=\left(x_{1}\left(t_{0}\right), x_{2}\left(t_{0}\right)\right)$ in $\mathbb{R}_{+}^{2}$ be the initial solution of system (3.3). By contradiction, suppose that there exists a solution $x(t)$ that lies outside of $\mathbb{R}_{+}^{2}$. The consequence is that $x(t)$ crosses the $x_{1}$ axis or $x_{2}$ axis. Now we have to consider two cases.

Case 1: If the solution $x(t)$ passes through the $x_{2}$ axis, then there exists $t^{*}$ such that $t^{*} \geq t_{0}$ and $x_{1}\left(t^{*}\right)=0$, and there exists $t_{1}$ sufficiently close to $t^{*}$ such that $t_{1}>t^{*}$ and $x_{1}(t)<0$ for all $t \in\left(t^{*}, t_{1}\right]$. By the previous conclusion there are two possibilities. 
(1i) If ${ }_{t_{0}}^{C} D_{t}^{\alpha} x_{1} \geq 0$ for all $t \in\left[t^{*}, t_{1}\right]$, based on the first equation of system (3.3), we obtain ${ }_{t_{0}}^{C} D_{t}^{\alpha} x_{1} \geq r x_{1}$. Using the Laplace transform in this inequality, the solution satisfies

$$
x_{1}(t) \geq x_{1}\left(t_{0}\right) E_{\alpha}\left(r\left(t-t_{0}\right)^{\alpha}\right), \quad t \in\left[t^{*}, t_{1}\right] .
$$

Since $x_{1}\left(t_{0}\right) \geq 0$, we have $x_{1}(t) \geq 0$, which contradicts the assumption. So $x_{1}(t) \geq 0$ for any $t \geq t_{0}$.

(1ii) If ${ }_{t_{0}}^{C} D_{t}^{\alpha} x_{1}<0$ for all $t \in\left[t^{*}, t_{1}\right]$, then

$$
\begin{aligned}
{ }_{t_{0}}^{C} D_{t}^{\alpha} x_{1} & =r_{1} x_{1}\left(1-\frac{e_{1}}{r_{1}}\right)-\frac{r_{1} x_{1}^{2}}{K_{1}+b_{12} x_{2}} \\
& =\left(r_{1}-e_{1}\right) x_{1}-\frac{r_{1} x_{1}^{2}}{K_{1}+b_{12} x_{2}} \\
& \geq\left(r_{1}-e_{1}\right) x_{1}-N x_{1}^{2}, \quad \text { where } N=\frac{r_{1}}{K_{1}}
\end{aligned}
$$

Since $t_{1}$ can be chosen to be arbitrarily close to $t^{*}$, then $x_{1}(t) \leq-x_{1}^{2}(t)$ for all $t \in\left[t^{*}, t_{1}\right]$. So, we obtain that

$$
{ }_{t_{0}}^{C} D_{t}^{\alpha} x_{1} \geq\left(r_{1}-e_{1}+N\right) x_{1}
$$

Using the Laplace transform in this inequality, we get that

$$
x_{1}(t) \geq x_{1}\left(t_{0}\right) E_{\alpha}\left(\left(r_{1}-e_{1}+N\right)\left(t-t_{0}\right)^{\alpha}\right), \quad t \in\left[t^{*}, t_{1}\right]
$$

Thus, $x_{1}(t) \geq 0$ for any $t \geq t_{0}$, which contradicts the assumption.

Case 2: Suppose that the solution $x(t)$ passes through the $x_{1}$ axis. Because the second equation of system (3.3) has the same form as the first one, the proof for case 2 is similar to the proof in the previous case.

Therefore, we can conclude that the solution $x(t)$ of system (3.3) lies within $\mathbb{R}_{+}^{2}$.

\section{Stability analysis of equilibrium points}

In this section, we investigate the stability of the equilibrium points by using the linearization method and the Lyapunov direct method. The equilibrium points of the model are obtained by solving the system of equations

$$
\left\{\begin{array}{l}
r_{1} x_{1}\left(1-\frac{e_{1}}{r_{1}}\right)-\frac{r_{1} x_{1}^{2}}{K_{1}+b_{12} x_{2}}=0 \\
r_{2} x_{2}\left(1-\frac{e_{2}}{r_{2}}\right)-\frac{r_{2} x_{2}^{2}}{K_{2}+b_{21} x_{1}}=0 .
\end{array}\right.
$$

We obtain four equilibrium points as follows:

1. The origin $E_{0}(0,0)$, which represents extinction of both species.

2. $E_{1}\left(K_{1} A_{1}, 0\right)$, where $A_{1}=1-\frac{e_{1}}{r_{1}}$, which represents extinction of the second species. The existence condition of $E_{1}$ is $0 \leq e_{1}<r_{1}$.

3. $E_{2}\left(0, K_{2} A_{2}\right)$, where $A_{2}=1-\frac{e_{2}}{r_{2}}$, which represents extinction of the first species. The existence condition of $E_{2}$ is $0 \leq e_{2}<r_{2}$.

4. $E_{3}\left(x_{1}^{*}, x_{2}^{*}\right)$, which is called the coexistence equilibrium point. 
As for the equilibrium points $E_{0}, E_{1}$, and $E_{2}$, they are called the noncoexistence equilibrium points. The existence condition of $E_{3}$ is presented in the next proposition.

Proposition 4.1 If

$$
A_{1} A_{2} b_{12} b_{21}<1, \quad 0 \leq e_{1}<r_{1} \text { and } 0 \leq e_{2}<r_{2} \text {, }
$$

then there exists a unique coexisting equilibrium $\left(x_{1}^{*}, x_{2}^{*}\right)$ of fractional-order system (3.3), where

$$
x_{1}^{*}=\frac{A_{1}\left(K_{1}+b_{12} A_{2} K_{2}\right)}{1-A_{1} A_{2} b_{12} b_{21}}, \quad x_{2}^{*}=\frac{A_{2}\left(K_{2}+b_{21} A_{1} K_{1}\right)}{1-A_{1} A_{2} b_{12} b_{21}} .
$$

Proof The proof for this proposition is the same as that for Proposition 1 of [54], which is the proof that there exists a unique coexisting equilibrium point for integer-order system (1.1).

\subsection{Local stability of equilibrium points}

In this subsection, we determine the local stability of the first three equilibrium points of system (3.3). The Jacobian matrix of the system is

$$
J\left(x_{1}, x_{2}\right)=\left(\begin{array}{cc}
r_{1}-e_{1}-\frac{2 r_{1} x_{1}}{K_{1}+b_{12} x_{2}} & \frac{b_{12} r_{1} x_{1}^{2}}{\left(K_{1}+b_{12} x_{2}\right)^{2}} \\
\frac{b_{21} r_{2} x_{2}^{2}}{\left(K_{2}+b_{21} x_{1}\right)^{2}} & r_{2}-e_{2}-\frac{2 r_{2} x_{2}}{K_{2}+b_{21} x_{1}}
\end{array}\right) .
$$

Theorem 4.2 If $r_{1}<e_{1}$ and $r_{2}<e_{2}$, then the trivial solution $E_{0}$ of the fractional-order system (3.3) is locally asymptotically stable.

Proof By (4.3) the Jacobian matrix $J\left(E_{0}\right)$ is as follows:

$$
J\left(E_{0}\right)=\left(\begin{array}{cc}
r_{1}-e_{1} & 0 \\
0 & r_{2}-e_{2}
\end{array}\right)
$$

The eigenvalues of $J\left(E_{0}\right)$ are $\lambda_{1}=r_{1}-e_{1}$ and $\lambda_{2}=r_{2}-e_{2}$. By the assumptions of the theorem, $\lambda_{1}<0$ and $\lambda_{2}<0$. Thus $\left|\arg \lambda_{1}\right|=\left|\arg \lambda_{2}\right|=\pi$. Therefore, according to Theorem 2.8, the equilibrium point $E_{0}$ is locally asymptotically stable.

Theorem 4.3 If $e_{1}<r_{1}$ and $r_{2}<e_{2}$, then the second species extinction equilibrium point $E_{1}\left(K_{1} A_{1}, 0\right)$ of the fractional-order system (3.3) is locally asymptotically stable.

Proof By (4.3) the Jacobian matrix $J\left(E_{1}\right)$ can be obtained as follows:

$$
J\left(E_{1}\right)=\left(\begin{array}{cc}
r_{1}\left(1-2 A_{1}\right)-e_{1} & b_{12} r_{1} A_{1}^{2} \\
0 & r_{2}-e_{2}
\end{array}\right) .
$$

Solving the characteristic equation $\operatorname{det}\left(\lambda I-J\left(E_{1}\right)\right)=0$ for $\lambda$ to find the eigenvalues of $J\left(E_{1}\right)$, we get

$$
\left(\lambda-r_{1}\left(1-2 A_{1}\right)+e_{1}\right)\left(\lambda-r_{2}+e_{2}\right)=0 .
$$


So, the eigenvalues of $J\left(E_{1}\right)$ are $\lambda_{3}=r_{1}\left(1-2 A_{1}\right)-e_{1}$ and $\lambda_{4}=r_{2}-e_{2}$. Since we assumed that $A_{1}=1-\frac{e_{1}}{r_{1}}, \lambda_{3}=e_{1}-r_{1}$, and $\lambda_{4}=r_{2}-e_{2}$. By the assumption it is clear that $\lambda_{3}<0$ and $\lambda_{4}<0$. According to Theorem 2.8 , the equilibrium point $E_{1}$ is locally asymptotically stable. This completes the proof.

Theorem 4.4 If $r_{1}<e_{1}$ and $e_{2}<r_{2}$, then the first species extinction equilibrium point $E_{2}\left(0, K_{2} A_{2}\right)$ of the fractional order system (3.3) is locally asymptotically stable.

Proof The idea of the proof is similar to that of Theorem 4.3.

\subsection{Global stability of positive coexistence equilibrium}

In this subsection, we investigate sufficient conditions for global uniform asymptotic stability of the positive coexisting equilibrium for the corresponding fractional-order system using the Lyapunov function.

Theorem 4.5 If $A_{1} b_{12}<2, A_{2} b_{21}<2, A_{1} A_{2} b_{12} b_{21}<1,0 \leq e_{1}<r_{1}$, and $0 \leq e_{2}<r_{2}$, then the unique interior positive equilibrium $\left(x_{1}^{*}, x_{2}^{*}\right)$ of the fractional-order system (3.3) is globally uniformly asymptotically stable on $\mathbb{R}_{+}^{2}$.

Proof We define the function $V_{1}:\left\{\left(x_{1}, x_{2}\right) \in \mathbb{R}_{+}^{2}: x_{1}>0, x_{2}>0\right\} \rightarrow \mathbb{R}$ by

$$
V_{1}\left(x_{1}, x_{2}\right)=c_{1} \int_{x_{1}^{*}}^{x_{1}} \frac{\theta-x_{1}^{*}}{K_{2}+b_{21} \theta} d \theta+c_{2} \int_{x_{2}^{*}}^{x_{2}} \frac{\theta-x_{2}^{*}}{K_{1}+b_{12} \theta} d \theta
$$

so that (4.6) can be written in the form

$$
\begin{aligned}
V_{1}\left(x_{1}, x_{2}\right)= & \frac{c_{1}}{b_{21}}\left(x_{1}-x_{1}^{*}-\left(\frac{K_{2}+b_{21} x_{1}^{*}}{b_{21}}\right) \ln \left(\frac{K_{2}+b_{21} x_{1}}{K_{2}+b_{21} x_{1}^{*}}\right)\right) \\
& +\frac{c_{2}}{b_{12}}\left(x_{2}-x_{2}^{*}-\left(\frac{K_{1}+b_{12} x_{2}^{*}}{b_{12}}\right) \ln \left(\frac{K_{1}+b_{12} x_{2}}{K_{1}+b_{12} x_{2}^{*}}\right)\right),
\end{aligned}
$$

where $c_{1}>0$ and $c_{2}=\frac{r_{1} b_{12} A_{1}}{r_{2} b_{21} A_{2}} c_{1}$. The function $V_{1}\left(x_{1}, x_{2}\right)$ is continuous on the domain $\mathbb{R}_{+}^{2}$, and $V_{1}\left(x_{1}, x_{2}\right)>0$ for all $\left(x_{1}, x_{2}\right) \in \mathbb{R}_{+}^{2} \backslash\left(x_{1}^{*}, x_{2}^{*}\right)$, whereas $V_{1}\left(x_{1}, x_{2}\right)=0$ if $x_{1}=x_{1}^{*}$ and $x_{2}=x_{2}^{*}$. Hence, we can see that $V_{1}\left(x_{1}, x_{2}\right)$ is a Lyapunov function. Also, $V_{1}\left(x_{1}, x_{2}\right)$ tends to $+\infty$ as either $x_{1}$ or $x_{2}$ tends to 0 or to $+\infty$. These properties mean that $V_{1}\left(x_{1}, x_{2}\right)$ is radially unbounded.

Applying the linearity property of the Caputo fractional derivative and using Theorem 2.6, we obtain

$$
\begin{aligned}
{ }_{t_{0}}^{C} D_{t}^{\alpha} V_{1}\left(x_{1}, x_{2}\right)= & { }_{t_{0}}^{C} D_{t}^{\alpha}\left(\frac{c_{1}}{b_{21}}\left(x_{1}-x_{1}^{*}-\left(\frac{K_{2}+b_{21} x_{1}^{*}}{b_{21}}\right) \ln \left(\frac{K_{2}+b_{21} x_{1}}{K_{2}+b_{21} x_{1}^{*}}\right)\right)\right) \\
& +{ }_{t_{0}}^{C} D_{t}^{\alpha}\left(\frac{c_{2}}{b_{12}}\left(x_{2}-x_{2}^{*}-\left(\frac{K_{1}+b_{12} x_{2}^{*}}{b_{12}}\right) \ln \left(\frac{K_{1}+b_{12} x_{2}}{K_{1}+b_{12} x_{2}^{*}}\right)\right)\right) \\
\leq & \frac{c_{1}}{b_{21}}\left({ }_{t_{0}}^{C} D_{t}^{\alpha} x_{1}-\left(\frac{K_{2}+b_{21} x_{1}^{*}}{K_{2}+b_{21} x_{1}}\right){ }_{t_{0}}^{C} D_{t}^{\alpha} x_{1}\right) \\
& +\frac{c_{2}}{b_{12}}\left({ }_{t_{0}}^{C} D_{t}^{\alpha} x_{2}-\left(\frac{K_{1}+b_{12} x_{2}^{*}}{K_{1}+b_{12} x_{2}}\right){ }_{t_{0}}^{C} D_{t}^{\alpha} x_{2}\right)
\end{aligned}
$$




$$
\begin{aligned}
= & \frac{c_{1}}{b_{21}}\left(1-\frac{K_{2}+b_{21} x_{1}^{*}}{K_{2}+b_{21} x_{1}}\right){ }_{t_{0}}^{C} D_{t}^{\alpha} x_{1} \\
& +\frac{c_{2}}{b_{12}}\left(1-\frac{K_{1}+b_{12} x_{2}^{*}}{K_{1}+b_{12} x_{2}}\right){ }_{t_{0}}^{C} D_{t}^{\alpha} x_{2} \\
= & \frac{c_{1}}{K_{2}+b_{21} x_{1}}\left(x_{1}-x_{1}^{*}\right)_{t_{0}}^{C} D_{t}^{\alpha} x_{1}+\frac{c_{2}}{K_{1}+b_{12} x_{2}}\left(x_{2}-x_{2}^{*}\right)_{t_{0}}^{C} D_{t}^{\alpha} x_{2} \\
= & \frac{c_{1}}{K_{2}+b_{21} x_{1}}\left(x_{1}-x_{1}^{*}\right)\left(r_{1} x_{1}\left(1-\frac{e_{1}}{r_{1}}\right)-\frac{r_{1} x_{1}^{2}}{K_{1}+b_{12} x_{2}}\right) \\
& +\frac{c_{2}}{K_{1}+b_{12} x_{2}}\left(x_{2}-x_{2}^{*}\right)\left(r_{2} x_{2}\left(1-\frac{e_{2}}{r_{2}}\right)-\frac{r_{2} x_{2}^{2}}{K_{2}+b_{21} x_{1}}\right) \\
= & \frac{c_{1} r_{1} x_{1}}{\left(K_{1}+b_{12} x_{2}\right)\left(K_{2}+b_{21} x_{1}\right)}\left(x_{1}-x_{1}^{*}\right)\left(A_{1} K_{1}+A_{1} b_{12} x_{2}-x_{1}\right) \\
& +\frac{c_{2} r_{2} x_{2}}{\left(K_{1}+b_{12} x_{2}\right)\left(K_{2}+b_{21} x_{1}\right)}\left(x_{2}-x_{2}^{*}\right)\left(A_{2} K_{2}+A_{2} b_{21} x_{1}-x_{2}\right) .
\end{aligned}
$$

By Proposition 4.1 the positive equilibrium point $\left(x_{1}^{*}, x_{2}^{*}\right)$ of system (3.3) satisfies the equalities

$$
A_{1} K_{1}=x_{1}^{*}-A_{1} b_{12} x_{2}^{*} \quad \text { and } \quad A_{2} K_{2}=x_{2}^{*}-A_{2} b_{21} x_{1}^{*} .
$$

We see that

$$
\begin{aligned}
{ }_{t_{0}}^{C} D_{t}^{\alpha} & V_{1}\left(x_{1}, x_{2}\right) \\
\leq & \frac{c_{1} r_{1} x_{1}}{\left(K_{1}+b_{12} x_{2}\right)\left(K_{2}+b_{21} x_{1}\right)}\left(x_{1}-x_{1}^{*}\right)\left(-\left(x_{1}-x_{1}^{*}\right)+A_{1} b_{12}\left(x_{2}-x_{2}^{*}\right)\right) \\
& +\frac{c_{2} r_{2} x_{2}}{\left(K_{1}+b_{12} x_{2}\right)\left(K_{2}+b_{21} x_{1}\right)}\left(x_{2}-x_{2}^{*}\right)\left(-\left(x_{2}-x_{2}^{*}\right)+A_{2} b_{21}\left(x_{1}-x_{1}^{*}\right)\right) .
\end{aligned}
$$

Since $c_{2}=\frac{r_{1} b_{12} A_{1}}{r_{2} b_{21} A_{2}} c_{1}$, we have

$$
\begin{aligned}
{ }_{t_{0}}^{C} D_{t}^{\alpha} & V_{1}\left(x_{1}, x_{2}\right) \\
\leq & \frac{c_{1} r_{1}}{\left(K_{1}+b_{12} x_{2}\right)\left(K_{2}+b_{21} x_{1}\right)}\left(-x_{1}\left(x_{1}-x_{1}^{*}\right)^{2}-x_{2} \frac{A_{1} b_{12}}{A_{2} b_{21}}\left(x_{2}-x_{2}^{*}\right)^{2}\right. \\
& \left.+A_{1} b_{12}\left(x_{1}+x_{2}\right)\left(x_{1}-x_{1}^{*}\right)\left(x_{2}-x_{2}^{*}\right)\right) \\
\leq & \frac{c_{1} r_{1}}{\left(K_{1}+b_{12} x_{2}\right)\left(K_{2}+b_{21} x_{1}\right)}\left(-x_{1}\left(x_{1}-x_{1}^{*}\right)^{2}-x_{2} \frac{A_{1} b_{12}}{A_{2} b_{21}}\left(x_{2}-x_{2}^{*}\right)^{2}\right. \\
& \left.+\frac{1}{2} A_{1} b_{12}\left(x_{1}+x_{2}\right)\left(x_{1}-x_{1}^{*}\right)^{2}+\frac{1}{2} A_{1} b_{12}\left(x_{1}+x_{2}\right)\left(x_{2}-x_{2}^{*}\right)^{2}\right) \\
= & \frac{-c_{1} r_{1}}{\left(K_{1}+b_{12} x_{2}\right)\left(K_{2}+b_{21} x_{1}\right)}\left(\left(x_{1}-\frac{1}{2} A_{1} b_{12}\left(x_{1}+x_{2}\right)\right)\left(x_{1}-x_{1}^{*}\right)^{2}\right. \\
& \left.+\left(x_{2} \frac{A_{1} b_{12}}{A_{2} b_{21}}-\frac{1}{2} A_{1} b_{12}\left(x_{1}+x_{2}\right)\right)\left(x_{2}-x_{2}^{*}\right)^{2}\right) .
\end{aligned}
$$


Let

$$
\begin{aligned}
W_{1}(x)= & \frac{c_{1} r_{1}}{\left(K_{1}+b_{12} x_{2}\right)\left(K_{2}+b_{21} x_{1}\right)}\left(\left(x_{1}-\frac{1}{2} A_{1} b_{12}\left(x_{1}+x_{2}\right)\right)\left(x_{1}-x_{1}^{*}\right)^{2}\right. \\
& \left.+\left(x_{2} \frac{A_{1} b_{12}}{A_{2} b_{21}}-\frac{1}{2} A_{1} b_{12}\left(x_{1}+x_{2}\right)\right)\left(x_{2}-x_{2}^{*}\right)^{2}\right) .
\end{aligned}
$$

We can verify that $W_{1}(x)$ is continuous on the domain $\mathbb{R}_{+}^{2}$ and $W_{1}(x)>0$ for all $x=\left(x_{1}, x_{2}\right) \in$ $\mathbb{R}_{+}^{2} \backslash\left(x_{1}^{*}, x_{2}^{*}\right)$, whereas $W_{1}(x)=0$ if $x_{1}=x_{1}^{*}$ and $x_{2}=x_{2}^{*}$. So, $W_{1}(x)$ is a positive definite function. Thus, ${ }_{t_{0}}^{C} D_{t}^{\alpha} V_{1}(t, x) \leq-W_{1}(x)$. By Theorem 2.9 the equilibrium point $\left(x_{1}^{*}, x_{2}^{*}\right)$ of system (3.3) is globally uniformly asymptotically stable in $\operatorname{int}\left(\mathbb{R}_{2}^{+}\right)$.

Remark 4.6 The Lyapunov function in Theorem 4.5 is modified from the function of integer-order differential systems presented in [14] and different from the functions presented in [45-48].

Remark 4.7 Theorem 4.5 can be applied to study a facultative mutualism of two species. In particular, the interaction between two species is assumed to be described by model (3.3) with parameters satisfying the conditions of the theorem. Subsequently, any solutions starting at a positive initial point eventually tend to the positive coexistence equilibrium of the model. This means biologically that the two species always coexist in the same habitat.

\section{Numerical simulations}

In this section, we present numerical examples to verify the theoretical results in Theorem 4.5 .

We carried out numerical simulations on the fractional-order two-species facultative mutualism system (3.3) to show the stability of the positive equilibrium result. The parameters $r_{1}=0.6, r_{2}=0.4, e_{1}=0.4, e_{2}=0.3, K_{1}=250, K_{2}=250, b_{12}=0.2$, and $b_{21}=0.25$ satisfy the assumptions of Theorem 4.5. Calculating the solution, we obtain the positive coexistence equilibrium point $x_{1}^{*}=87.8661$ and $x_{2}^{*}=67.9916$.

Many numerical methods have been applied to solve nonlinear fractional differential equations such as the Adams method [55], the predictor corrector method [56], the Adomian decomposition method [57], the extrapolation method [58], and the variational iteration method [59]. However, among these methods, the Adams method is useful for studying the long-term behavior of the solutions of nonlinear fractional differential equations [60]. Thus, in this study, we use the Adams method for solving the model with the Matlab software.

The numerical solution of system (3.3) is presented in Figures 1 and 2. In Figure 1, we fix $\alpha=0.5$ and choose three different initial conditions as follows: $\left(x_{1}(0), x_{2}(0)\right)=$ $(70,110),(90,90),(110,70)$. In Figure 2 , we fix the initial condition $\left(x_{1}(0), x_{2}(0)\right)=(110,70)$ and choose various values of $\alpha=0.3,0.5$, and 0.7. Figure 1(1a) and Figure 2(2a) show the relationship between the values of the species $x_{1}, x_{2}$ with respect to times $t$. Figure 1(1b) and Figure 2(2b) present descriptions of the dynamic behavior of two interacting species.

The parameters in the model satisfy all the conditions of Theorem 4.5. Hence, the equilibrium point $\left(x_{1}^{*}, x_{2}^{*}\right)=(87.8661,67.9916)$ is globally uniformly asymptotically stable on $\mathbb{R}_{2}^{+}$. From Figures 1 and 2 we can see that all the solutions of the system converge uniformly to this point. 


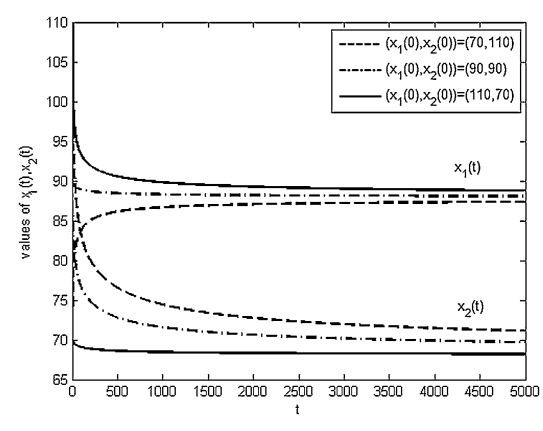

(1a)

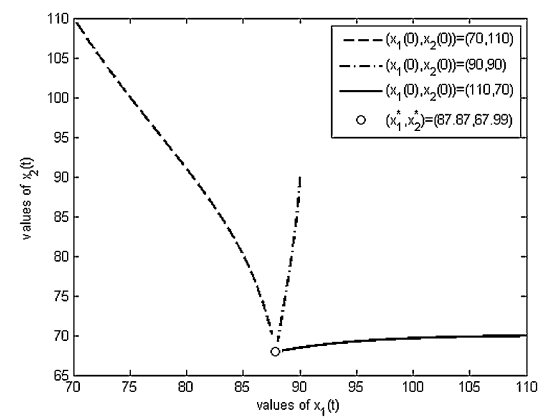

(1b)

Figure 1 (1a) The relationship between the numbers of individuals of species $x_{1}, x_{2}$ with respect to time $t$. (1b) The relationship between the values of $x_{1}$ and $x_{2}$ with $\alpha=0.5$ for $\left(x_{1}(0), x_{2}(0)\right)=(70,110)$, $(90,90)$, and $(110,70)$.

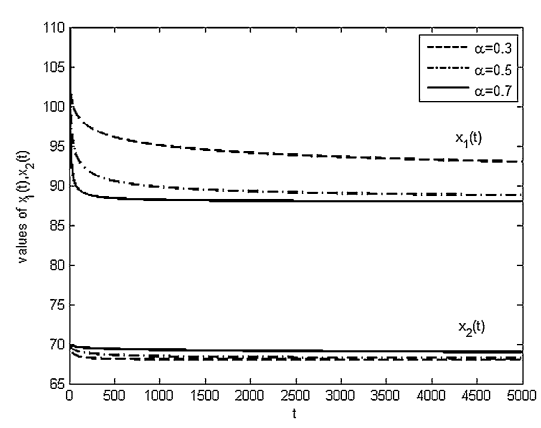

(2a)

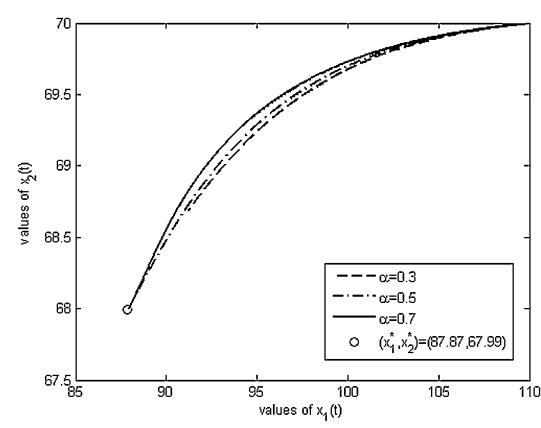

(2b)

Figure 2 (2a) The relationship between the numbers of individuals of species $x_{1}, x_{2}$ with respect to times $t$. (2b) The relationship between the values of $x_{1}$ and $x_{2}$ with $\left(x_{1}(0), x_{2}(0)\right)=(110,70)$ for $\alpha=0.3$, 0.5 , and 0.7 .

\section{Conclusion}

In this study, we presented a Caputo fractional-order model of two-species facultative mutualism and analyzed the stability of the model. We investigated the local asymptotic stability of the three noncoexistence equilibrium points using the linearization method. For the coexistence equilibrium point, we analyzed the global uniform asymptotic stability via the Lyapunov method. These results provide sufficient conditions to ensure the local asymptotic stability and global uniform asymptotic stability of the model. Finally, we used some numerical simulations of the model to illustrate the stability results.

\section{Acknowledgements}

This research is supported by Department of Mathematics, Faculty of Science, Chiang Mai University. Appreciation is extended toward the Centre of Excellence in Mathematics, CHE, for their financial support.

Competing interests

The authors declare that they have no competing interests.

Authors' contributions

Both authors read and approved the final manuscript.

\section{Author details}

'Department of Mathematics, Faculty of Science, Chiang Mai University, Chiang Mai, 50200, Thailand. ${ }^{2}$ Center of Excellence in Mathematics and Applied Mathematics, Department of Mathematics, Faculty of Science, Chiang Mai 
University, Chiang Mai, 50200, Thailand. ${ }^{3}$ Centre of Excellence in Mathematics, CHE, 328 Si Ayutthaya Road, Bangkok, Thailand.

\section{Publisher's Note}

Springer Nature remains neutral with regard to jurisdictional claims in published maps and institutional affiliations.

Received: 10 August 2017 Accepted: 20 November 2017 Published online: 28 November 2017

\section{References}

1. Brauer, F, Castillo-Chavez, C: Mathematical Models in Population Biology and Epidemiology, vol. 40. Springer, Berlin (2001)

2. Fulford, G, Forrester, P, Jones, A: Modelling with Differential and Difference Equations, vol. 10. Cambridge University Press, Cambridge (1997)

3. Kuang, Y: Delay Differential Equations: With Applications in Population Dynamics, vol. 191. Academic Press, San Diego (1993)

4. Salisbury, A: Mathematical models in population dynamics. Dissertation. New College of Florida (2011)

5. Vanmeter, KC, Hubert, RJ: Microbiology for the Healthcare Professional, 2nd edn. Mosby Elsevier, Amsterdam (2015)

6. Stachowicz, JJ, Hay, ME: Facultative mutualism between an herbivorous crab and a coralline alga: advantages of eating noxious seaweeds. Oecologia 105(3), 377-387 (1996)

7. Rockwood, LL: Introduction to Population Ecology. Blackwell, Oxford (2006)

8. Gilbert, LE: Ecological consequences of a coevolved mutualism between butterflies and plants. In: Coevolution of Animals and Plants, pp. 210-240. University of Texas Press, Austin (1980)

9. Handel, SN: The competitive relationship of three woodland sedges and its bearing on the evolution of ant-dispersal of Carex pedunculata. Evolution 32(1), 151-163 (1978)

10. Batra, LR: Insect-fungus symbiosis: nutrition, mutualism, and commensalism. In: International Mycological Congress (2nd: 27 Aug.-3 Set. 1977), Florida (1979)

11. Roughgarden, J: Evolution of marine symbiosis - a simple cost-benefit model. Ecology 56(5), 1201-1208 (1975)

12. Legovic, T, Gecek, S: Impact of maximum sustainable yield on mutualistic communities. Ecol. Model. 230, 63-72 (2012)

13. Wolin, C, Lawlor, L: Models of facultative mutualism: density effects. Am. Nat. 124, 843-862 (1984)

14. Georgescu, P, Zhang, H: Lyapunov functional for two-species mutualisms. Appl. Math. Comput. 229, 754-764 (2014)

15. Igor, P: Fractional Differential Equations. Mathematics in Science and Engineering, vol. 198. Academic Press, New York (1999)

16. Caputo, M: Linear models of dissipation whose Q almost frequency independent: II (reprint). Fract. Calc. Appl. Anal. 11(1), 3-14 (2008)

17. Luo, Y, Chen, YQ: Fractional Order Motion Controls. Wiley, Chichester (2012)

18. Machado, JT, Kiryakova, V, Mainardi, F: Recent history of fractional calculus. Commun. Nonlinear Sci. Numer. Simul. 16(3), 1140-1153 (2011)

19. Almeida, R, Bastos, NRO, Monteiro, MT: Modeling some real phenomena by fractional differential equations. Math. Methods Appl. Sci. 39(16), 4846-4855 (2016)

20. Demirci, E: A fractional order model for obesity epidemic in a non-constant population. Adv. Differ. Equ. 2017, 79 (2017)

21. Area, I, Batarfi, H, Losada, J, Nieto, JJ, Shammakh, W, Torres, Á: On a fractional order Ebola epidemic model. Adv. Differ. Equ. 2015(1), 278 (2015)

22. Zafar, ZUA, Rehan, K, Mushtaq, M: Fractional-order scheme for bovine babesiosis disease and tick populations. Adv. Differ. Equ. 2017(1), 86 (2017)

23. Mairet, F, Ramírez, H, Rojas-Palma, A: Modelling and stability analysis of a microalgal pond with nitrification. Anual Sociedad de Matemática de Chile 51, 448-468 (2015)

24. Menouer, MA, Moussaoui, A, Dads, EA: Existence and global asymptotic stability of positive almost periodic solution for a predator-prey system in an artificial lake. Chaos Solitons Fractals 103, 271-278 (2017)

25. Lu, G, Lu, Z: Geometric approach to global asymptotic stability for the SEIRS models in epidemiology. Nonlinear Anal., Real World Appl. 36, 20-43 (2017)

26. Guo, L, Chen, YQ: System stability analysis via a perturbation technique. Commun. Nonlinear Sci. Numer. Simul. 57, 111-124 (2017)

27. Berezansky, L, Braverman, E: A note on stability of Mackey-Glass equations with two delays. J. Math. Anal. Appl. 450(2), 1208-1228 (2017)

28. Hou, Q, Wang, T: Global stability and a comparison of SVEIP and delayed SVIP epidemic models with indirect transmission. Commun. Nonlinear Sci. Numer. Simul. 43, 271-281 (2017)

29. Caetano, D, Faria, T: Stability and attractivity for Nicholson systems with time-dependent delays. Electron. J. Qual. Theory Differ. Equ. 2017, 63 (2017)

30. Liu, B: Asymptotic behavior of solutions to a class of non-autonomous delay differential equations. J. Math. Anal. Appl. 446(1), 580-590 (2017)

31. Baleanu, D, Wu, GC, Zeng, SD: Chaos analysis and asymptotic stability of generalized Caputo fractional differential equations. Chaos Solitons Fractals 102, 99-105 (2017)

32. Čermák, J, Došlá, Z, Kisela, T: Fractional differential equations with a constant delay: stability and asymptotics of solutions. Appl. Math. Comput. 298, 336-350 (2017)

33. Yadav, VK, Das, S, Bhadauria, BS, Singh, AK, Srivastava, M: Stability analysis, chaos control of a fractional order chaotic chemical reactor system and its function projective synchronization with parametric uncertainties. Chin. J. Phys. 55(3), 594-605 (2017)

34. Ji, G, Ge, Q, Xu, J: Dynamic behavior of a fractional order two-species cooperative systems with harvesting. Chaos Solitons Fractals 92, 51-55 (2016) 
35. Huo, J, Zhao, H: Dynamical analysis of a fractional SIR model with birth and death on heterogeneous complex networks. Phys. A, Stat. Mech. Appl. 448, 41-56 (2016)

36. Ghaziani, RK, Alidousti, J, Eshkaftaki, AB: Stability and dynamics of a fractional order Leslie-Gower prey-predator model. Appl. Math. Model. 40(3), 2075-2086 (2016)

37. Li, Y, Chen, YQ, Igor, P: Stability of fractional-order nonlinear dynamic systems: Lyapunov direct method and generalized Mittag-Leffler stability. Comput. Math. Appl. 59(5), 1810-1821 (2010)

38. Baranowski, J, Zagorowska, M, Bauer, W, Dziwinski, T, Piatek, P: Applications of direct Lyapunov method in Caputo non-integer order systems. Elektron. Elektrotech. 21(2), 10-13 (2015)

39. Li, C, Ma, Y: Fractional dynamical system and its linearization theorem. Nonlinear Dyn. 71(4), 621-633 (2013)

40. Li, Y, Chen, YQ, Igor, P: Mittag-Leffler stability of fractional order nonlinear dynamic systems. Automatica 45(8), 1965-1969 (2009)

41. Zhang, F, Li, C, Chen, YQ: Asymptotical stability of nonlinear fractional differential system with Caputo derivative. Int. J. Differ. Equ. 2011, 12 (2011)

42. Delavari, H, Baleanu, D, Sadati, J: Stability analysis of Caputo fractional-order nonlinear systems revisited. Nonlinear Dyn. 67(4), 2433-2439 (2012)

43. Gallegos, JA, Duarte-Mermoud, MA: On the Lyapunov theory for fractional order systems. Appl. Math. Comput. 287, 161-170 (2016)

44. Liu, S, Jiang, W, Li, X, Zhou, XF: Lyapunov stability analysis of fractional nonlinear systems. Appl. Math. Lett. 51, 13-19 (2016)

45. Vargas-De-León, C: Volterra-type Lyapunov functions for fractional-order epidemic systems. Commun. Nonlinear Sci. Numer. Simul. 24(1-3), 75-85 (2015)

46. Duarte-Mermoud, MA, Aguila-Camacho, N, Gallegos, JA, Castro-Linares, R: Using general quadratic Lyapunov functions to prove Lyapunov uniform stability for fractional order systems. Commun. Nonlinear Sci. Numer. Simul. 22(1), 650-659 (2015)

47. Aguila-Camacho, N, Duarte-Mermoud, MA, Gallegos, JA: Lyapunov functions for fractional order systems. Commun. Nonlinear Sci. Numer. Simul. 19(9), 2951-2957 (2014)

48. Zhou, XF, Hu, LG, Liu, S, Jiang, W: Stability criterion for a class of nonlinear fractional differential systems. Appl. Math. Lett. 28, 25-29 (2014)

49. Liang, S, Wu, R, Chen, L: Laplace transform of fractional order differential equations. Electron. J. Differ. Equ. 2015(139), 1 (2015)

50. Kexue, L, Jigen, P: Laplace transform and fractional differential equations. Appl. Math. Lett. 24(12), 2019-2023 (2011)

51. Abdeljawad, T, Gejji, V: Lyapunov-Krasovskii stability theorem for fractional systems with delay. Rom. J. Phys. 56(5-6), 636-643 (2011)

52. Ahmed, E, El-Sayed, AMA, El-Saka, HAA: On some Routh-Hurwitz conditions for fractional order differential equations and their applications in Lorenz, Rössler, Chua and Chen systems. Phys. Lett. A 358(1), 1-4 (2006)

53. Walter, W: Ordinary Differential Equations. Springer Graduate Texts in Mathematics, vol. 182 (1991)

54. Vargas-De-León, C: Lyapunov functions for two-species cooperative systems. Appl. Math. Comput. 219(5), 2493-2497 (2012)

55. Diethelm, K, Ford, NJ, Freed, AD: Detailed error analysis for a fractional Adams method. Numer. Algorithms 36(1), 31-52 (2004)

56. Diethelm, K, Ford, NJ, Freed, AD: A predictor-corrector approach for the numerical solution of fractional differential equations. Nonlinear Dyn. 29(1), 3-22 (2002)

57. Adomian, G: Solving Frontier Problems of Physics: The Decomposition Method, vol. 60. Springer, Berlin (2013)

58. Diethelm, K, Walz, G: Numerical solution of fractional order differential equations by extrapolation. Numer. Algorithms 16(3), 231-253 (1997)

59. Wu, G, Lee, EWM: Fractional variational iteration method and its application. Phys. Lett. A 374(25), 2506-2509 (2010)

60. Deshpande, AS, Daftardar-Gejji, V, Sukale, YV: On Hopf bifurcation in fractional dynamical systems. Chaos Solitons Fractals 98, 189-198 (2017)

\section{Submit your manuscript to a SpringerOpen ${ }^{\circ}$ journal and benefit from:}

- Convenient online submission

- Rigorous peer review

- Open access: articles freely available online

- High visibility within the field

- Retaining the copyright to your article

Submit your next manuscript at $\gg$ springeropen.com 